\title{
Distância Ótima para Instalação de Armadilhas Atrativas para Captura de Vespas Sociais Neotropicais: uma Projeção Estatística
}

\author{
Tatiane Tagliatti Maciel $^{1 *}$, Bruno Corrêa Barbosa ${ }^{1} \&$ Fábio Prezoto $^{1}$ \\ ${ }^{1}$ Laboratório de Ecologia Comportamental e Bioacústica, Departamento de Zoologia, Instituto de Ciências Biológicas, Universidade Federal de Juiz. de \\ Fora, Juiz de Fora, MG, Brasil. \\ *E-mail para correspondência: tatitagliatti@hotmail.com
}

\begin{abstract}
RESUMO
Tendo em vista a importância de inventários de fauna e a falta de estudos dedicados aos atributos espaciais das espécies de vespas sociais, ou ainda dos métodos utilizados para sua coleta, este estudo estima e analisa em escala local, a distância ótima para instalação de armadilhas atrativas para estudos de diversidade de vespas sociais. O trabalho foi desenvolvido no Jardim Botânico da Universidade Federal de Juiz de Fora, onde foram instaladas 40 armadilhas atrativas a fim de avaliar distâncias de instalação entre $10 \mathrm{~m}$ e $390 \mathrm{~m}$ umas das outras. A partir dos resultados, é possível sugerir que, de acordo com o tamanho da área estudada, as armadilhas sejam instaladas a distâncias entre $10 \mathrm{~m}$ e $150 \mathrm{~m}$ umas das outras, possibilitando um menor número de armadilhas em campo e poupando tempo e custo.
\end{abstract}

Palavras-chave: Amostragem, Atividade de forrageio, Diversidade, Raio de ação, Vespidae.

\section{ABSTRACT}

Optimum distance for installing attractive neotropical social wasp traps: a statistical projection. Considering the value of fauna inventories and the lack of studies dedicated to the spatial aspects of social wasp species, or the methods used to collect them, this study estimates and analyzes, on a local scale, the ideal distance for the installation of attractive traps for studies social wasp diversity studies. The work was developed at the Botanic Garden of the Federal University of Juiz de Fora, in which 40 attractive traps were installed to evaluate the installation distances between $10 \mathrm{~m}$ and $390 \mathrm{~m}$ from each other. From the results, it is possible to suggest that, according to the size of the studied area, the traps are installed at distances between $10 \mathrm{~m}$ and $150 \mathrm{~m}$ from others, allowing fewer traps in the field and saving time and cost.

Keywords: Diversity, Flight range, Foraging activity, Sampling, Vespidae. 
Para a coleta de vespas sociais (Hymenoptera: Polistinae) em uma determinada área, o método mais utilizado é o de armadilhas atrativas, ou seja, são instaladas armadilhas iscadas com algum recurso alimentar, como carboidrato e / ou proteína, que atraem e capturam esses insetos no momento em que estão forrageando (Souza et al., 2015; Barbosa et al., 2016). Neste método, assim como acontece na natureza com frutos maduros e restos de animais, por exemplo, cada armadilha libera substâncias voláteis provenientes da fermentação das iscas num determinado raio (Pareja et al., 2007; Somavilla et al., 2019; Maciel, 2014), atraindo assim as vespas sociais.

A polinização e o controle biológico de pragas, que correspondem às principais funções ecológicas de interesse humano exercidas pelas vespas sociais, são realizados através do comportamento de forrageio (Clemente et al., 2012; Prezoto et al., 2019). A distância percorrida pelas vespas sociais durante sua atividade de forrageio está diretamente relacionada à disponibilidade de recurso no ambiente, ou seja, quanto menor a quantidade de recursos maior será a distância que as vespas precisarão percorrer (Prezoto et al., 2016).

Na literatura não existem estudos que avaliem o raio de atração das armadilhas atrativas, no entanto, em trabalhos de diversidade que utilizam esse método, ainda que sem justificativa, há uma padronização quanto à distância de instalação das armadilhas a 10 m umas das outras (Maciel et al., 2016). É importante destacar que essa distância pode implicar na reamostragem de indivíduos de uma mesma colônia visto que o raio de ação de vespas sociais pode chegar a $150 \mathrm{~m}$ (Prezoto et al., 2016), subestimando assim a diversidade de uma área.

Visto que os estudos atuais somente aplicam a metodologia de coleta de armadilhas atrativas já apresentada na literatura, este estudo vai além e estima e analisa em escala local a distância ótima para instalação de armadilhas atrativas para estudos de diversidade de vespas sociais.

A experimentação foi conduzida no Jardim Botânico da Universidade Federal de Juiz de Fora (JB - UFJF), que está localizado no perímetro urbano de Juiz de Fora ( $21^{\circ} 43^{\prime} 28^{\prime \prime} \mathrm{S}-43^{\circ} 16^{\prime} 47^{\prime \prime} 0 ; 800 \mathrm{~m}$ a.s.I), sudeste do estado de Minas Gerais, Brasil, a 750 m de altitude e apresenta clima subtropical quente com inverno seco e verão chuvoso (Cwa) (Sá-Júnior et al., 2012). O trabalho foi realizado em uma trilha pré-existente no JB - UFJF denominada "Palmital" (Oliveira-Neto, 2014), onde há uma predominância de palmito-juçara [Euterpe edulis Mart. (Aracaceae)].

Foram instaladas 40 armadilhas atrativas que, com base na literatura, foram iscadas com suco de frutas, cerveja ou vinho tinto (Maciel et al., 2016) e, como proposto por Souza et al. (2015), equidistantes a $10 \mathrm{~m}$ e a uma altura de 1,5 $\mathrm{m}$ do solo. As armadilhas permaneceram em campo por cinco dias consecutivos ao longo de 12 campanhas realizadas entre novembro de 2015 e maio de 2016. Cada 
armadilha correspondeu a um ponto de amostragem onde foram registradas a riqueza e a abundância de vespas sociais. Por etapas, pontos intermediários foram desconsiderados a fim de obter distâncias de instalação entre 10 m e 390 m.

As espécies foram identificadas pelas chaves propostas por Richards (1978), Hermes e Kohler (2004), e Gelin (2009). Alguns espécimes foram montados em via seca para compor a caixa testemunha depositada no Laboratório de Ecologia Comportamental e Bioacústica (LABEC) da Universidade Federal de Juiz de Fora.

Utilizando a distância padrão de $10 \mathrm{~m}$ para avaliar se houve diferença estatística entre a riqueza e a abundância de vespas sociais registradas por todas as distâncias, foi utilizado o teste não paramétrico para duas amostras independentes de Mann-Whitney, gerado pelo programa BioEstat 5.3 (Ayres et al., 2007).

No total, o estudou registrou oito espécies de vespas sociais: Agelaia multipicta (Haliday, 1836), Agelaia vicina (Saussure, 1854), Polybia fastidiosuscula Saussure, 1854, Polybia jurinei Saussure, 1854, Polybia lugubris Ducke, 1905, Polybia platycephala Richards, 1951, Polistes pacificus pacificus Fabricius 1804 e Polistes versicolor (Olivier, 1791). Quando comparadas à distância padrão de 10 m, as armadilhas instaladas a até $40 \mathrm{~m}$ umas das outras não apresentaram diferença significativa quanto à abundância de espécies coletadas (ver Tabela 1); ao avaliar a riqueza de espécies, nenhuma distância apresentou diferença significativa (ver Tabela 1), no entanto, armadilhas instaladas a até $150 \mathrm{~m}$ registraram riqueza de $100 \%$ das espécies amostradas e, acima de $150 \mathrm{~m}$, a riqueza se manteve abaixo de $75 \%$ do que foi amostrado.

A diferença na abundância pode ser justificada pelo fato de que quanto menos armadilhas forem instaladas, menor será o número de indivíduos coletados. Por outro lado, por se tratarem de pontos independentes de coleta, o número de espécies se mantém constante, corroborando ainda os resultados encontrados por Silveira et al. (2012) e Maciel et al. (2016), que sugerem que a determinação da fauna de uma área não é definida pelo método de coleta e sim pela fitofisionomia do local.

Na literatura, a instalação das armadilhas atrativas a uma distância de $10 \mathrm{~m}$ umas das outras é padronizada, no entanto, nunca foi justificada. Assim, dos 21 trabalhos de diversidade realizados em Minas Gerais (ver Maciel et al., 2016), apenas Freitas et al. (2015) utilizaram 40 m, que mesmo sem justificar a escolha por essa distância de instalação, foi corroborado pelos resultados do presente estudo em relação à abundância de espécies.

A partir de uma revisão na literatura Togni (2009) concluiu que o raio médio de forrageio das vespas sociais comumente capturadas por armadilhas atrativas (Epiponini) é de 93,75 $\pm 56,04 \mathrm{~m}$, corroborando que uma distância de apenas $10 \mathrm{~m}$ entre as armadilhas pode subestimar a diversidade do local e que 
distâncias a partir de 40 m podem ser mais eficientes. Assim, sabendo-se que se trata de uma estimativa estatística e considerando que em campo as armadilhas podem influenciar a capacidade de atração de armadilhas vizinhas, é possível sugerir que armadilhas instaladas a uma distância de até 150 m umas das outras, a depender do tamanho da área estudada, são efetivas em registrar a diversidade da área. Além disso, com o aumento da distância de instalação entre as armadilhas, com o mesmo número de armadilhas utilizadas antes (com instalação a $10 \mathrm{~m}$ uma das outras), o pesquisador poderá aumentar a área amostrada mantendo o mesmo esforço de instalação e o mesmo gasto com as iscas. Por outro lado, se o tamanho da área for mantido, haverá uma diminuição no número de armadilhas.

Tabela 1: Resultado do teste de Mann-Whittney (U; $p$-valor) para riqueza e abundância de vespas sociais registradas por armadilhas atrativas instaladas a distâncias entre $10 \mathrm{~m}$ e $390 \mathrm{~m}$ no Jardim Botânico da UFJF, Juiz de Fora, MG.

\begin{tabular}{|c|c|c|c|c|c|c|c|}
\hline Distância & $10 \mathrm{~m} \times 20 \mathrm{~m}$ & $10 \mathrm{~m} \times 30 \mathrm{~m}$ & $10 \mathrm{~m} \times 40 \mathrm{~m}$ & $10 \mathrm{~m} \times 50 \mathrm{~m}$ & $10 \mathrm{~m} \times 60 \mathrm{~m}$ & $10 \mathrm{~m} \times 70 \mathrm{~m}$ & $10 \mathrm{~m} \mathrm{X80m}$ \\
\hline \multirow{2}{*}{ Abundância } & $U=1.1552$ & $U=1.3653$ & $U=1.9954$ & $U=1.7328$ & $U=2.3105$ & $U=2.2054$ & $U=2.6255$ \\
\hline & $p=0.2480$ & $p=0.1722$ & $p=0.0460$ & $p=0.0831$ & $p=0.0209$ & $p=0.0274$ & $p=0.0087$ \\
\hline \multirow{2}{*}{ Riqueza } & $U=0.8402$ & $U=0.0000$ & $U=0.8402$ & $U=0.0000$ & $U=0.8402$ & $U=0.8402$ & $U=1.2603$ \\
\hline & $p=0.4008$ & $p=1.0000$ & $p=0.4008$ & $p=1.0000$ & $p=0.4008$ & $p=0.4008$ & $p=0.2076$ \\
\hline \multirow{3}{*}{$\begin{array}{l}\text { Distância } \\
\text { Abundância }\end{array}$} & $10 \mathrm{~m} \times 90 \mathrm{~m}$ & $10 \mathrm{~m} \times 100 \mathrm{~m}$ & $10 \mathrm{~m} \times 110 \mathrm{~m}$ & $10 \mathrm{~m} \times 120 \mathrm{~m}$ & $10 \mathrm{~m} \times 130 \mathrm{~m}$ & $10 \mathrm{~m} \times 140 \mathrm{~m}$ & $10 \mathrm{~m} \times 150 \mathrm{~m}$ \\
\hline & $U=2.1529$ & $U=2.2580$ & $U=2.6780$ & $U=2.6780$ & $U=2.3630$ & $U=2.6780$ & $U=2.4680$ \\
\hline & $p=0.0313$ & $p=0.0239$ & $p=0.0074$ & $p=0.0074$ & $p=0.0181$ & $p=0.0074$ & $p=0.0136$ \\
\hline \multirow{2}{*}{ Riqueza } & $U=1.6803$ & $U=0.8402$ & $U=1.6803$ & $U=1.2603$ & $U=0.4201$ & $U=1.6803$ & $U=0.4201$ \\
\hline & $p=0.0929$ & $p=0.4008$ & $p=0.0929$ & $p=0.2076$ & $p=0.6744$ & $p=0.0929$ & $p=0.6744$ \\
\hline \multirow{3}{*}{$\begin{array}{l}\text { Distância } \\
\text { Abundância }\end{array}$} & $10 \mathrm{~m} \times 160 \mathrm{~m}$ & $10 \mathrm{~m} \times 170 \mathrm{~m}$ & $10 \mathrm{~m} \times 180 \mathrm{~m}$ & $10 \mathrm{~m} \times 190 \mathrm{~m}$ & $10 \mathrm{~m} \times 200 \mathrm{~m}$ & $10 \mathrm{~m} \times 210 \mathrm{~m}$ & $10 \mathrm{~m} \times 220 \mathrm{~m}$ \\
\hline & $U=2.6255$ & $U=2.1529$ & $U=2.6780$ & $U=2.5205$ & $U=2.7305$ & $U=2.7831$ & $U=2.8356$ \\
\hline & $p=0.0087$ & $p=0.0313$ & $p=0.0074$ & $p=0.0117$ & $p=0.0063$ & $p=0.0054$ & $p=0.0046$ \\
\hline \multirow{2}{*}{ Riqueza } & $U=1.2603$ & $U=1.2603$ & $U=1.6803$ & $U=1.2603$ & $U=0.8402$ & $U=1.2603$ & $U=1.6803$ \\
\hline & $p=0.2076$ & $p=0.2076$ & $p=0.0929$ & $p=0.2076$ & $p=0.4008$ & $p=0.2076$ & $p=0.0929$ \\
\hline \multirow{3}{*}{$\begin{array}{l}\text { Distância } \\
\text { Abundância }\end{array}$} & $10 \mathrm{~m} \times 230 \mathrm{~m}$ & $10 \mathrm{~m} \times 240 \mathrm{~m}$ & $10 \mathrm{~m} \times 250 \mathrm{~m}$ & $10 \mathrm{~m} \times 260 \mathrm{~m}$ & $10 \mathrm{~m} \times 270 \mathrm{~m}$ & $10 \mathrm{~m} \times 280 \mathrm{~m}$ & $10 \mathrm{~m} \times 290 \mathrm{~m}$ \\
\hline & $U=2.7831$ & $U=2.9406$ & $U=2.6780$ & $U=2.6780$ & $U=2.3630$ & $U=2.6780$ & $U=2.7831$ \\
\hline & $p=0.0054$ & $p=0.0033$ & $p=0.0074$ & $p=0.0074$ & $p=0.0181$ & $p=0.0074$ & $p=0.0054$ \\
\hline \multirow{2}{*}{ Riqueza } & $U=1.2603$ & $U=1.6803$ & $U=1.2603$ & $U=1.2603$ & $U=1.6803$ & $U=1.6803$ & $U=1.6803$ \\
\hline & $p=0.2076$ & $p=0.0929$ & $p=0.2076$ & $p=0.2076$ & $p=0.0929$ & $p=0.0929$ & $p=0.0929$ \\
\hline Distância & $10 \mathrm{~m} \times 300 \mathrm{~m}$ & $10 \mathrm{~m} \times 310 \mathrm{~m}$ & $10 \mathrm{~m} \times 320 \mathrm{~m}$ & $10 \mathrm{~m} \times 330 \mathrm{~m}$ & $10 \mathrm{~m} \times 340 \mathrm{~m}$ & $10 \mathrm{~m} \times 350 \mathrm{~m}$ & $10 \mathrm{~m} \times 360 \mathrm{~m}$ \\
\hline \multirow{2}{*}{ Abundância } & $U=2.6780$ & $U=2.7831$ & $U=2.7305$ & $U=2.7831$ & $U=2.3630$ & $U=2.3630$ & $U=2.8356$ \\
\hline & $p=0.0074$ & $p=0.0054$ & $p=0.0063$ & $p=0.0054$ & $p=0.0181$ & $p=0.0181$ & $p=0.0046$ \\
\hline \multirow{2}{*}{ Riqueza } & $U=1.2603$ & $U=1.6803$ & $U=1.2603$ & $U=1.6803$ & $U=1.6803$ & $U=1.6803$ & $U=1.6803$ \\
\hline & $p=0.2076$ & $p=0.0929$ & $p=0.2076$ & $p=0.0929$ & $p=0.0929$ & $p=0.0929$ & $p=0.0929$ \\
\hline Distância & $10 \mathrm{~m} \times 370 \mathrm{~m}$ & $10 \mathrm{~m} \times 380 \mathrm{~m}$ & $10 \mathrm{~m} \times 390 \mathrm{~m}$ & & & & \\
\hline \multirow{2}{*}{ Abundância } & $U=2.5205$ & $U=2.6780$ & $U=2.6780$ & & & & \\
\hline & $p=0.0117$ & $p=0.0074$ & $p=0.0074$ & & & & \\
\hline \multirow{2}{*}{ Riqueza } & $U=1.6803$ & $U=1.2603$ & $U=0.8402$ & & & & \\
\hline & $p=0.0929$ & $p=0.2076$ & $p=0.4008$ & & & & \\
\hline
\end{tabular}




\section{AGRADECIMENTOS}

Os autores gostariam de agradecer à Tropical Indústria de Alimentos (TIAL) pela doação de todos os sucos utilizados no trabalho, e ao apoio da Universidade Federal de Juiz de Fora (UFJF), da Coordenação de Aperfeiçoamento de Pessoal de Nível Superior (CAPES), do Conselho de Desenvolvimento Científico e Tecnológico (CNPq) e da Fundação de Amparo à Pesquisa do Estado de Minas Gerais (FAPEMIG).

\section{REFERÊNCIAS BIBLIOGRÁFICAS}

Ayres, M.; Ayres Júnior, M.; Ayres, D.L. \& Santos, A.D.A.D. 2007. BioEstat: Aplicações Estatísticas nas Áreas das Ciências Biomédicas. ONG Mamiraua. Belém, PA.

Barbosa, B.C.; Detoni, M.; Maciel, T.T. \& Prezoto, F. 2016. Studies of social wasp diversity in Brazil: Over 30 years of research, advancements and priorities. Sociobiology 63: 858-880.

Clemente, M.A.; Lange, D.; Del-Claro, K.; Prezoto, F.; Campos, N.R. \& Barbosa, B.C. 2012. Flower-visiting social wasps and plants interaction: Network pattern and environmental complexity. Psyche: A Journal of Entomology 2012: 1-10.

Freitas, J.L.; Pires E.P.; De Oliveira T.T.C.; Dos Santos N.L. \& De Souza M.M. 2015. Vespas sociais (Hymenoptera: Vespidae) em lavouras de Coffea arabica L. (Rubiaceae) no Sul de Minas Gerais. Revista Agrogeoambiental 7: 69-79.

Gelin, L.F.F. 2009. Análise Filogenética de Polybia Lepeletier, 1836 (Hymenoptera, Vespidae, Polistinae). Dissertação (Mestrado em Biologia Animal). Instituto de Biociências, Letras e Ciências Exatas (IBILCE) São José do Rio Preto, SP. 111p.

Hermes, M.G. \& Köhler A. 2004. The Genus Agelaia Lepeletier (Hymenoptera, Vespidae, Polistinae) in Rio Grande do Sul, Brazil. Revista Brasileira de Entomologia 48: 135-138.

Maciel, T.T. 2014. Atratividade de Iscas na Captura de Lepidópteros Frugívoros em Mata Atlântica. Trabalho de Conclusão do Curso (Ciências Biológicas). Centro de Ensino Superior de Juiz de Fora, Juiz de Fora, MG. 23p.

Maciel, T.T.; Barbosa, B.C. \& Prezoto F. 2016. Armadilhas Atrativas como Ferramenta de Amostragem de Vespas Sociais (Hymenoptera: Vespidae): Uma Meta-Análise. EntomoBrasilis 9: 150-157.

Oliveira-Neto, N.E. 2014. Aspectos Ecológicos da Comunidade Arbórea em um Trecho de Floresta 
Atlântica com Predominância de Palmito-juçara (Euterpe edulis Martius). Dissertação (Mestrado em Ecologia). Universidade Federal de Juiz de Fora, Juiz de Fora, MG. 77p.

Pareja, M.; Moraes, M.V.B.; Clark, S.J.M.; Birkett, A. \& Powell, W. 2007. Response of the aphid parasitoid Aphidius funebris to volatiles from undamaged and aphid-infested Centaurea nigra. Journal of Chemical Ecology 33: 695-710.

Prezoto, F.; Barbosa, B.C.; Maciel, T.T. \& Detoni, M. 2016. Agroecossistemas e o serviço ecológico dos insetos na sustentabilidade. In: Resende, L.O.; Prezoto, F., Barbosa B.C. \& Gonçalves, E.L. (eds.). Sustentabilidade: Tópicos da Zona da Mata Mineira. 1 ed. Juiz de Fora, Real Consultoria em Negócios Ltda. 74p.

Prezoto, F.; Maciel, T.T.; Detoni, M.; Mayorquin, A.Z. \& Barbosa, B.C. 2019. Pest Control Potential of Social Wasps in Small Farms and Urban Gardens. Insects 10(7): 1-10.

Richards, O.W. 1978. The Social Wasps of the Americas Excluding the Vespinae. London, British Museum (Natural History). 580p.

Sá Júnior, A.; Carvalho L.G.; Silva F.F. \& Carvalho A.M. 2012. Application of the Köppen classification for climatic zoning in the state of Minas Gerais, Brazil. Theoretical and Applied Climatology 108: 1-7.

Silveira, O.T.; Silva, S.S.; Pereira, J.L.G. \& Tavares, I.S. 2012. Local-scale spatial variation in diversity of social wasps in an Amazonian rain forest, Caxiuanã, Pará, Brazil (Hymenoptera, Vespidae, Polistinae). Revista Brasileira de Entomologia 56: 329-346.

Somavilla, A.; Souza, J.L.P.; da Silva, A.O. \& Keppler, R.L.F. 2019. Occurrence of Hymenoptera on pig carcasses in a tropical rainforest in Central Amazonia, Brazil. Sociobiology 66(2): 389-393.

Souza, M.M.; Perillo, L.N.; Barbosa, B.C. \& Prezoto, F. 2015. Use of flight interception traps of Malaise type and attractive traps for social wasps record (Vespidae: Polistinae). Sociobiology 62: 450-456.

Togni, O.C. 2009. Diversidade de Vespas Sociais (Hymenoptera, Vespidae) na Mata Atlântica do Litoral Norte do Estado de São Paulo. Trabalho de Dissertação (Ciências Biológicas). Universidade Estadual Paulista, Rio Claro, SP. 99p. 